
\title{
25 Research Soure \\ B-Cell Gene Expression and Microbiota Prior Immunization Profile Vaccine Humoral Responsiveness
}

\section{Elena Gonçalves}

Sorbonne Universite https://orcid.org/0000-0002-0474-0677

\section{Yolanda Guillén}

IrsiCaixa AIDS Research Institute-HIVACAT

Javier R Lama

Asociacion Civil Impacta Salud y Educacion

Jorge Sanchez

Universidad Nacional Mayor de San Marcos

Christian Brander

IrsiCaixa AIDS Research Institute-HIVACAT

\section{Roger Paredes}

Infectious Diseases Department, Hospital Universitari Germans Trias

Behazine Combadière ( $\sim$ behazine.combadiere@inserm.fr)

https://orcid.org/0000-0002-2353-4406

\section{Research}

Keywords: biomarkers, vaccination, transcriptomic, microbiota, neutralizing antibodies, systems biology

Posted Date: July 23rd, 2020

DOl: https://doi.org/10.21203/rs.3.rs-46833/v1

License: (c) (i) This work is licensed under a Creative Commons Attribution 4.0 International License.

Read Full License 


\section{Abstract}

Background: The identification of new biomarkers is essential to making it possible to predict the degree of protection following vaccination. Very few human studies have focused on baseline characteristics including microbiota and gene expression to underlie vaccine immune responsiveness. We investigated the host whole-blood transcriptome and microbiome before vaccination, to assess the likelihood of their involvement in an effective MVA-neutralizing antibody response (MVA-Nab) two months later.

Results: We based our analyses on data obtained from a randomized clinical study in which participants $(n=10)$ were vaccinated with the MVA-HIV clade B vaccine (MVA-B). Samples were collected at 2-time points prior vaccination (week-2, w0) to study blood transcriptome. Skin wrap (site of vaccination) and stool microbiota were analysed for diversity and abundance (16S rRNAseq). MVA-neutralizing antibody responses were measured at week 8 . The levels of MVA-Nab responses were positively correlated with an abundance of Eubacterium in stool and Prevotella in skin. The simultaneous investigation of blood transcriptome and host microbiota before vaccination showed that genus diversity and bacterial abundance at that time correlated significantly with the expression of genes involved in B cell development stages. The combination of gene expression and microbiota makes it possible to forecast strong responders to MVA-B vaccination.

Conclusion: To our knowledge, this is the first study integrating host blood gene expression and microbiota before vaccination to predict the intensity of humoral response months later. The genes identified are involved in B cell differentiation might open an avenue of research in this field to optimize vaccination strategies.

\section{Background}

The largest fraction of immune cells is found at sites colonized by microorganisms, such as the skin or the gastrointestinal $(\mathrm{GI})$ tract [1]. The gut microbiome is essential for the development, maturation, and adequate functioning of the immune system $[1,2]$. The human skin is colonized during the postnatal period by microorganisms that prevent the invasion of external pathogens. Crosstalk between these commensals and the immune system is necessary to trigger innate and adaptive immune responses. Increased attention to the relation between the gut commensal bacteria and host immune responses has led scientists to question whether these microorganisms affect the efficacy of vaccines $[3,4]$. Moreover, although the composition of fecal microbiota may be one of the multiple factors that modulate host responses to external immunization, little is known about its role in the interindividual disparity in vaccine efficacy.

The first observation of a potential link between the microbiome and vaccines occurred in an oral vaccination model that used a heat-labile enterotoxin from Escherichia coli as an adjuvant $[5,6]$. In that situation, depletion of the intestinal microbiota was associated with a profound depression of antigenspecific Th1 and Th17 lymphocytes. Similarly, high antibody responses to the seasonal trivalent 
influenza vaccine (TIV) and polio vaccine (IPOL) require the presence of intestinal commensals [7]. Inversely, impaired in microbiota composition and diversity have been reported to attenuate immune responses to vaccines [8]. Interestingly, in human infants receiving hepatitis $B$, diphtheria, tetanus, and Haemophilus influenza type B vaccines, a randomized placebo-controlled double-blind trial demonstrated that vaccine-specific immune responses were enhanced by probiotics $[9,10]$. Recently described crossreactivity between gut microbiota antigens and naive and memory $C D 4^{+} T$ and $B$ cells [11] suggests that the antibody response to HIV-1 immunization may be shaped by intestinal B cells stimulated by host commensals [12]. The microbiota is known to be required for a mature B-cell compartment [13]. For example, germ-free mice have abnormalities in their B-cell systems and lower IgA levels than colonized animals of the same genotype [14]. Microbial antigens and microbial metabolites, such as short-chain fatty acids, strongly promote plasma cell differentiation at mucosal and systemic sites [15]. These microbial metabolites promote IgA production by regulating the metabolism and gene expression in $B$ cells in mice models and in in vitro study of human B cells $[15,16]$. This IgA appears to orchestrate the beneficial mutualism established between the host and gut commensal microbiome by interacting directly with microbiota species.

Presentation of microbial antigens by the different MHC genotypes also contributes to modifying the $\lg \mathrm{A}$ repertoires, which in turn modulate the composition of the microbiota in the gut [17]. Accordingly, the depletion of anti-inflammatory microbial species and an expansion of proinflammatory species have been observed in human selective IgA deficiency [18]. A lack of intestinal microbial stimulation results in fewer $\lg A^{+}$plasma cells in the gut and a lower abundance of IgA in mouse models [19-21]. Thus the diversity of IgA on the mammalian intestinal surface matches the intestinal taxa diversity [22]. For these reasons, host microbial profiling during vaccine administration to might help optimize the vaccine responses and improve the tolerability of multiple antipathogen treatments. The microbiota, after all, constitutes a constant source of natural adjuvants capable of activating a multitude of pathways that control innate and adaptive immunity [23].

Systems biology has been successfully used to investigate the fundamental innate immune mechanisms orchestrating protective adaptive responses after the perturbation of vaccination against yellow fever [24, 25], HIV [26], Ebola [27], and influenza [28]. An important challenge, however, is to analyze individual baseline human health characteristics to help identify those at higher risk of infection despite vaccination. Until now, only a few studies have looked for candidate traits associated with vaccine responsiveness and partially predicting the humoral response to vaccination against influenza [29-32]. No study has examined the interrelations between each individual's immunological state, their microbiota at baseline, and the impact of both on their vaccine-induced immune responses. As the most successful vaccines act through the production of antibodies [33], identifying specific individual characteristics at baseline should enhance our ability for dividing vaccinees into "high responders" or "low responders" [34]. Such predictive markers might serve as a potential diagnostic tool that assists vaccine development by taking into account the interindividual heterogeneity of immune responses. 
This study used a systems biology approach to investigate the volunteers' immune predisposition to respond to MVA-B vaccination, assessed by their blood transcriptome profile; specifically that related to their $\mathrm{B}$ cell differentiation stages, and its conditioning by the human microbiota before vaccination. That is, we investigated the host gene expression in blood by a microarray approach and the skin and stool microbiota by using $16 \mathrm{~S}$ ribosomal RNA sequencing both before vaccination. The objective was to examine their potential involvement in an effective MVA-B neutralizing antibody (Nabs) response during the CUTHIVAC 03 randomized phase Ib clinical study. This trial immunized $10 \mathrm{HIV}$ seronegative subjects aged from 18 to 45 years by the intramuscular route with MVA-HIV clade B vaccine. We analyzed their baseline transcriptomic signature and baseline bacterial abundance and diversity in skin and stool to assess their potential association with the intensity of the Nabs response.

\section{Material And Methods}

\section{Skin and feces sampling}

For each individual, skin swab samples from the deltoid muscle region $(\sim 5-20 \mathrm{~cm}$ below the vaccine administration site) were collected before the vaccination (w0). Skin samples were collected with CatchAll ${ }^{\text {TM }}$ Sample Collection Swab kits moistened with SCF-1 solution. The skin surface was sampled for 30 seconds by firmly swabbing the cotton tip back and forth $\sim 50$ times. The cotton tip was stored in sterile tubes with MoBio solution at $-80^{\circ} \mathrm{C}$ until DNA extraction. Fecal samples for each participant were collected in sterile fecal collection tubes the day before the vaccination, matching the skin sample time points. All samples were stored at $4-5^{\circ} \mathrm{C}$ until their reception at the IMPACTA clinical trial site, where they were cryopreserved at $-80^{\circ} \mathrm{C}$. All samples were shipped on dry ice to the IrsiCaixa AIDS Research Institute for DNA extraction, amplification, and sequencing.

\section{DNA extraction and amplicon sequencing from skin and fecal samples}

DNA extraction was performed with the DNA Extraction kit from Epicentre Technologies $\subseteq$ (Madison, WI, USA). Six aliquots of buffer solution from the DNA extraction kit were used as negative controls. To amplify the variable V3-V4 region from the 16S rRNA gene, we used the primer pair described in the MiSeq $^{\text {TM }}$ rRNA Amplicon Sequencing protocol, which already has the Illumina adapter overhang nucleotide sequences added to the 16S rRNA V3-V4-specific primers, i.e., 16S_F 5'-( TCG GCA GCG TCA GAT GTG TAT AAG AGA CAG CCT ACG GGN GGC WGC AG)-3' and 16S_R 5'-(GTC TCG TGG GCT CGG AGA TGT GTA TAA GAG ACA GGA CTA CHV GGG TAT CTA ATC C) -3'. Amplifications were performed in triplicate $25-\mu \mathrm{L}$ reactions, each containing $2.5 \mu \mathrm{L}$ of non-diluted DNA template, $12.5 \mu \mathrm{L}$ of KAPA HiFi HotStart Ready Mix (containing KAPA HiFi HotStart DNA Polymerase, buffer, $\mathrm{MgCl} 2$, and dNTPs, KAPA Biosystems Inc., Wilmington, MA, USA), and $5 \mu \mathrm{L}$ of each primer at $1 \mu \mathrm{M}$. Thermal cycling conditions consisted of an initial denaturation step $\left(3\right.$ minutes at $95^{\circ} \mathrm{C}$ ), followed by 30 cycles of denaturation ( 30 seconds at $95^{\circ} \mathrm{C}$ ), annealing $\left(30\right.$ seconds at $55^{\circ} \mathrm{C}$ ), and extension (30 seconds at $72^{\circ} \mathrm{C}$ ). These were followed by a final extension step of 10 minutes at $72{ }^{\circ} \mathrm{C}$. Once the desired amplicon was confirmed in $1 \%$ agarose gel 
electrophoresis, all three replicates were pooled and stored at $-30{ }^{\circ} \mathrm{C}$ until the sequencing library was prepared. After amplified DNA templates were cleaned up for non-DNA molecules and Illumina sequencing adapters and dual indices attached with the Nextera XT Index Kit (Illumina, Inc.), the corresponding PCR amplification program was run, as described in the MiSeq 16S rRNA Amplicon Sequencing protocol. After a second round of cleanup, amplicons were quantified with the Quant-iT ${ }^{T M}$ PicoGreen ${ }^{\circledR}$ dsDNA Assay Kit (Invitrogen, Carlsbad, MA, USA) and diluted in equimolar concentrations ( $4 \mathrm{nM}$ ) for further pooling. Sequencing was performed on an Illumina MiSeq ${ }^{\mathrm{TM}}$ platform (Illumina, Inc.) according to the manufacturer's specifications to generate a median of 30,644 paired-end sequences of $\sim 300$ bp length in each direction ( 61,289 reads per sample).

\section{Sequence quality control and microbiota analyses}

The quality of MiSeq raw sequences was assessed with the FastQC software [35] (http://www.bioinformatics.babraham.ac.uk/projects/fastqc/). Sequences were trimmed with Trimmommatic [36], with a cutoff value of Q30 for both ends, a minimum mean threshold of Q20 for 30bp-sliding window across sequences, and a minimum read length of $250 \mathrm{bp}$ (Supplemental Figs. 1a and b). After quality control, 28 samples including controls $(n=8)$ and volunteers $(n=10,5$ women and 5 men) for skin and stools, were further analyzed. Mothur pipeline [37] was used to bin 16S rDNA sequences into operational taxonomic units (OTUs) with a threshold of $97 \%$ sequence similarity. OTUs present in only a single sample were discarded. Rarefaction curves were represented by defining the maximum subsampling size as the number of sequences of the sample with the fewest sequences (2751 sequences for skin samples, and 1059 sequences for stool samples) (Supplemental Fig. 1c). Richness and diversity indexes were estimated by using the summary.single module implemented in mothur. For taxonomical analysis, 16S rDNA sequences were classified according to the GreenGenes database [38] version 13.5.99.

\section{MVA-GFP Neutralizing antibody assay}

Anti-MVA neutralizing activities were evaluated in serum collected at week 8 (w8) with an assay based on GFP detection by flow cytometry [39, 40]. It used HeLa cells as targets and a recombinant strain of MVA expressing the enhanced Aequoriae GFP [41]. Serial dilutions of heat inactivated serum were performed in 96-well round-bottom tissue culture plates (TPP, Zurich, Switzerland) containing DMEM (Gibco, Invitrogen) supplemented with $2 \%$ fetal calf serum (PAA, Laboratories $\mathrm{GmbH}$, Pashing, Austria). MVAeGFP was then added to each well at a MOI of 0.25 . The plate was then incubated for 1 hour at $37^{\circ} \mathrm{C}$ until the addition of $1 \times 10^{5} \mathrm{HeLa}$ cells. The incubation then continued for an additional 16 hours at $37^{\circ} \mathrm{C}$, $0.5 \% \mathrm{CO}_{2}$. After trypsinization, the cells were washed with PBS supplemented with $0.5 \%$ fetal calf serum and 2 mM EDTA and fixed with $2 \%$ formaldehyde. GFP expression was analyzed with FACSCanto II and Diva software (BD Biosciences). The percentage of neutralization was defined as the ratio of the reduction in the number of GFP-expressing cells to the number of GFP-expressing cells in untreated control wells. 


\section{RNA extraction and data preprocessing for transcriptomic analysis}

Whole blood samples of $2.5 \mathrm{~mL}$ were collected in PAXgene RNA tubes (PreAnalytix) twice from each volunteer two weeks before ( $w-2)$ and the day of the vaccination (w0). These tubes enable the preservation and stabilization of RNA (storage at $-80^{\circ} \mathrm{C}$ ). Total RNA was extracted from whole blood according to the instructions in the handbook accompanying the PAXgene blood RNA Kit (PreAnalytiX, Hombrechtikon, Switzerland). RNA purity and integrity were assessed on the Agilent 2100 Bioanalyzer with the RNA 6000 Nano LabChip reagent set (Agilent, Palo Alto, CA, USA). Samples for microarray hybridization were prepared as described in the Affymetrix GeneChip WT PLUS Reagent Kit User Manual (Affymetrix, Inc., Santa Clara, CA, USA). For hybridization (to Affymetrix Human Gene 2.1 ST Array Plates), washing, staining, and scanning took place in an Affymetrix GeneTitan system, controlled by the Affymetrix GeneChip Command Console software w4.2. Background signal correction was performed by applying the backgroundCorrect function from the limma package on the perfect match (PM) signals with R Software 3.3.1. The underlying model is the normal-exponential convolution model from RMA (chip intensity: addition of a signal exponentially distributed, chip noise: follows Gaussian distribution) [42]. The variance stabilizing transformation algorithm (justvsn function from the vsn package [43] was applied to the background corrected signal (monotonic transformation), and the signal then transformed back to its usual scale by exponentiation (base 2). To make the chips comparable, a quantile normalization [44] (normalize function from the affy package) was then applied to the variance-stabilized signal. The probe signals for replicated arrays were averaged and a quantile normalization performed anew. In all, 24,768 probes were analyzed.

\section{Statistical analyses}

Microbiome samples were clustered according to their genus composition by a nonmetric multidimensional scaling (NMDS) approach based on ecological distance matrices calculated by BrayCurtis dissimilarities, as implemented in $\mathrm{R}$ (Vegan, metaMDS, and ggplot2 packages). NMDS ellipses were drawn based on a confidence interval $(\mathrm{Cl})$ of 0.95 . To determine significant factors that describe the community structure better, we used a multivariate ADONIS test with terms added sequentially. The associations between baseline genus abundance or genus diversity, blood gene expression, and MVA-Nab response were evaluated by using the Spearman rank correlation test with significance defined by $P$ value $<0.05$. The heatmap was performed with values row-centered and scaled, Pearson correlation as the distance method and a dendrogram computed and reordered based on row means. The heatmap, logistic regression analyses, and ROC curves were performed and generated with R. Ingenuity ${ }^{\circledR}$ pathway analysis $(\mathrm{IPA} \otimes)$ was used to perform functional enrichment analyses and identify new targets or candidate biomarkers within the context of biological systems. It provided the canonical pathways, molecular/cellular functions, and networks that were statistically overrepresented in the gene signatures.

\section{Ethics and community involvement}


The study was conducted in accordance with the Declaration of Helsinki and the International Conference on Harmonization Good Clinical Practice guidelines and approved by the relevant regulatory and independent ethics committees. Each participant provided written informed consent before study entry. The study was registered and approved by the Peru regulatory authorities (IMPACTA IRB 0037-2014-CE; Peru NIH 396-2014-OG-OGITT-OPE/INS).

\section{Results}

\section{Study of host microbiota before vaccination and relation to post-vaccination humoral responses}

The study included five men and five women (18-45 years old) vaccinated by the intramuscular route to assess the safety and immunogenicity of MVA-HIV clade B (MVA-B), results reported elsewhere (Sanchez \& Goncalves, manuscript submitted). Exploratory analysis of whole blood samples at two distinct timepoints before vaccination ( $\mathrm{w}-2$ and $\mathrm{w} 0$ ) studied the gene expression profile and the skin and stool samples for microbiome analysis (w0) at baseline. As expected, the microbial composition differed between the skin and stool samples (Supplemental Fig. 2a-c). In addition, the stool samples showed dissimilarities between men and women, but this comparison did not reach statistically significant differences $(P<0.097)$ (Supplemental Fig. 2a). The predominant microbial families relatively abundant in skin samples were Moraxellaceae, Staphylococcaceae and Pseudomonadaceae, whereas Ruminococcaceae, Lachnospiraceae, Prevotellaceae, and Bacteroidaceae were predominant in stool samples (Supplemental Fig. 2c). The 16S RNA sequencing generated several metrics: richness (sobs: number of observed OTUs; chao: Chao1 richness estimate; ace: Abundance-based coverage estimation) and diversity (Shannon: Shannon diversity index; sd_invsimpson: inverse Simpson diversity index). The amplitude of the humoral response was defined by the MVA-specific IgG neutralizing antibodies measured in serum at w8 post-vaccination. We observed no correlation between the MVA-Nab response and the baseline indexes of diversity and richness in either skin or stool (data not shown). We did however find significant positive correlations between the abundance of both skin Prevotella $(r=0.76, P=$ 0.0159) (Fig. 1a) and fecal Eubacterium ( $r=0.68, P=0.0351)$ (Fig. 1b) at baseline with MVA-Nab response.

\section{Whole blood gene expression and host microbiota before vaccination are associated with post-vaccination humoral responses}

To improve our understanding of host molecular mechanisms potentially associated with skin and gut microbiota that may be involved in vaccine immunogenicity, we counted the number of genes at baselines that were correlated with the MVA-Nab response at w8. We confirmed that gene expression of the baseline samples did not differ between w-2 and w0 using hierarchal clustering analysis (data not 
shown). Out of all samples, we found 154 significant genes correlated with the MVA-Nab response ( $P<$ 0.05) (Fig. 2a). However, no correlation was observed between genus diversity and MVA-Nab response.

Next, we looked for a correlation between the microbiota diversity index and the genes $(n=154)$ correlated at baseline with MVA-Nab responses. We found 22 genes for skin and 19 for stool that were correlated with at least one diversity index (Shannon or sd_invsimpson) (Fig. 2a, Supplemental Tables 1 and 2), including 10 common genes to the skin and stool samples. Among these genes, we observed one gene cluster positively correlated with MVA-Nab response and another negatively correlated with humoral response (Fig. 2a). According to the IPA analysis, the negatively correlated genes appear to be involved in protein transmembrane transport, translation and transcription regulation, cell division, migration, proliferation, and differentiation, as well as in the oxidation reduction and metabolic processes. The positively correlated genes, on the other hand, appeared involved in cell homeostasis and migration, cell growth, proliferation, regulation of gene expression, the apoptotic process, exocytosis, and intracellular signal transduction. Interestingly, among the 10 common genes to the skin and stool samples we found the IGLV8-61, BLK, and EBF1 genes which are involved in antigen recognition, B cell development, proliferation, and differentiation, and in the positive regulation of transcription in B cell and B cell receptor signaling (Fig. 2a). Surprisingly these three significant genes involved in B cell development stages were negatively correlated with the baseline abundance of Prevotella and Eubacterium, respectively for skin and stool (Fig. 2b). To assess the predictive power of this signature of three genes and each of the two microbial genera, we ran logistic regression models (Fig. 2c). Use of the expression of the three genes and Prevotella abundance in the skin microbiota has an $85.42 \%$ chance, assessed by its area under the curve, of correctly predicting MVA-Nab responders, while with the three-gene signature and Eubacterium abundance in the stool microbiota there is an $89.58 \%$ chance of correctly predicting MVA-Nab responders (Fig. 2c). These results suggest that advanced B lymphocyte differentiation before vaccination, potentially signaled by high expression of these three genes, and associated with low abundance of Prevotella or Eubacterium, is associated with poor MVA-Nab response.

\section{Discussion}

To our knowledge, this work is the first to investigate the crosstalk between pre-vaccination host gene expression in blood cells, skin and stool microbiota and their association with the intensity of ensuing post-vaccination Nab responses. The data may provide important guidance for future design and refinement of vaccine strategies aiming at the induction of neutralizing antibody-mediated immunity. The limitation of this study is the small number of individuals included. However, the strength of our work is the availability of two sets of gene expression data collected at baseline $(w-2, w 0)$ that is often absent in other studies. It is intriguing to discover three genes, all involved in B cell differentiation and proliferation correlated with humoral responses 2 months later. Further validation studies are necessary in the future.

First, we observed that the abundance of particular skin or stool bacteria were associated with the MVA$\mathrm{Nab}$ response. Abundant Prevotella in the skin at baseline was positively correlated with MVA-Nab response. Prevotella is known to promote mucosal inflammation and to stimulate production of epithelial 
cell cytokines [45]. Prevotella is also found in larger numbers in the skin of women aged 60-76 years than in that of women in their $20 \mathrm{~s}$ and $30 \mathrm{~s}$ and was enriched in all of the skin sites of the older group compared to the younger ones [46]. In stool, we found that Eubacterium abundance at baseline was positively correlated with the MVA-Nab response. This family of bacteria is known to be associated with gut health [47-49], and several of its species are higher in centenarians than in either young or elderly adults [50]. The potential impact of the gut microbiota on vaccine immunogenicity has been already investigated with systemic vaccines [51] and with oral vaccines including those of rotavirus (RVV), polio, and cholera, mainly in infants/children living in low-income countries $[52,53]$. For example, bacteria related to Streptococcus bovis species were more abundant before vaccination in Ghanaian vaccineresponders than non-responders and were positively associated with RVV efficacy, whereas Bacteroides and Prevotella species were more common in the microbiome of nonresponders and correlated with a lack of RVV response [54]. In Bangladeshi infants, the pre-vaccination presence of Bifidobacterium was positively associated with some adaptive immunological responses, such as $\mathrm{CD} 4^{+}$and $\mathrm{CD} 8^{+} \mathrm{T}$-cell proliferative responses to BCG and tetanus toxoid vaccinations as well as specific lgG responses to tetanus toxoid and hepatitis $B$ vaccines, whereas high levels of enteric pathogens such as Enterobacteriales and Pseudomonadales were associated with neutrophilia and poorer vaccine responses [51].

Secondly, we examined the pre-vaccination host blood genes that were correlated with MVA-Nab intensity. We then investigated microbiota abundance to decipher a minimal gene signature predictive of MVA-Nab responsiveness. Interestingly, within this signature we find BLK, IGLV8-61 and EBF1 involved in B cell development, proliferation and differentiation and in the positive regulation of transcription in $\mathrm{B}$ cells and $B$ cell receptor signaling. The BLK gene belongs to the family of protein tyrosine kinases src, and the $B$ cells activation induces BLK gene product phosphorylation playing a key role in transmitting signals through surface immunoglobulins which supports the pro-B to pre-B transition and the signaling for growth arrest and apoptosis downstream of B-cell receptor [55]. BLK also plays a role in the development, differentiation, and activation of B cells and in the intracellular signaling pathway. BLK is detected in pro$B$ cells and persists in mature $B$ cells but is absent in plasma cells. Triple protein tyrosine kinase (SFK)deficient mice - BLK, LYN, and FYN - have impaired NFkB signaling and B cell development [56]. EBF1, an early $B$ cell factor 1 , is one of the transcription factors essential for orchestrating the development of the $B$ cell line. Heterozygosity of EBF1 results in the deregulation of at least eight transcription factors involved in lymphopoiesis and the deregulation of key proteins that play a crucial role in the survival, development, and differentiation of pro-B cells [57]. IGLV8 (variable domain) is a glycoprotein produced by $B$ lymphocytes; its binding of a specific antigen triggers the clonal expansion and differentiation of $B$ lymphocytes into immunoglobulin-secreting plasma cells. The link between microbiota and host blood transcriptome has also been studied previously by Nakaya et al., who showed that TLR5 expression in blood 3 days after influenza vaccination was correlated with antibody response 28 days later [28]. This correlation was significantly lower in TLR5-deficient mice immunized with TIV compared to wild-type mice. As influenza vaccine does not stimulate TLR5 directly, however, Oh et al. demonstrated with germ- 
free or antibiotic-treatment that the commensal bacteria were the source of the TLR5 ligands responsible for enhancing immune response to TIV [7].

\section{Conclusion}

It should be noted that in our study the three genes were negatively correlated with MVA-Nab response and microbial diversity of both skin and stool samples but also with the abundance of the Prevotella family in skin and the Eubacterium family in stool. The logistic regression based on the expression of these three genes and Prevotella and Eubacterium abundance for, respectively, skin and stool, highlights the predictive power of this signature for the MVA-Nab immune responses. These results propose that an advanced differentiation state of B lymphocytes before vaccination, potentially represented by a high expression of these three genes and associated with low genus abundance and diversity, might be associated with poor MVA-Nab response.

\section{Declarations}

\section{Ethics approval and consent to participate}

The study was conducted in accordance with the Declaration of Helsinki and the International Conference on Harmonization Good Clinical Practice guidelines and approved by the relevant regulatory and independent ethics committees. Each participant provided written informed consent before study entry. The study was registered and approved by the Peru regulatory authorities (IMPACTA IRB 0037-2014-CE; Peru NIH 396-2014-OG-OGITT-OPE/INS).

\section{Consent for publication}

Not applicable

\section{Availability of data and materials}

Raw Illumina MiSeq sequences and study metadata were deposited in the National Center for Biotechnology Information - NCBI repository (Bioproject accession number: XXXXXXXX, SRA accession number: $X X X X X X)$.

\section{Competing interest}

CB is co-founder, shareholder and employee of Aelix Therapeutics, outside of this work.

\section{Funding}


This project has received funding from the European Union's Horizon 2020 research and innovation programme under grant agreement No. 681137 and support by the Fondation Dormeur, Vaduz, (Liechtenstein). RP and CB were partly funded from the European Union's Horizon 2020 Research and Innovation programme under the grant agreement no 847943 (MISTRAL).

\section{Author contribution}

Conceptualization (BC, $C B)$, Methodology (BC, $C B, J S, R P)$, Funding (BC, $C B, J S, R P)$, Acquisition and validation ( $E G, Y G)$, Formal Analysis ( $E G, Y G, B C)$, Investigation ( $E G, Y G, B C, R P)$, Supervision ( $B C, C B, J S$, $J L, R P)$, Resources (BC, CB, JS, JL, RP), Data curation (EG, YG) - Writing (EG, YG, BC) - Original Draft preparation Writing ( $E G, Y G)$ - Review Editing (all authors), Visualization (EG, YG), Project Administration (BC, CB, JS, RP).

\section{Acknowledgment}

We thank members of the CUTHIVAC-EU consortium and Cuthivac Clinical study group : Pedro Gonzáles, María Fernández-Maldonado, Susana Perez, Samandhy Cedeño, Annika Vogt, Marco Antonio Fernández, Olivia bonduelle, Eric pedruzzi, Mariona Parera and Marc Noguera-Julián, Carmela Ganoza, Beatriz Mothe, Carmen E. Gòmez, Mariano Esteban, Felipe Garcia. We also thank Julien Nourikyan, Simon de Bernard from Altrabio, Lyon, France and Jo Ann Cahn for editing the English of the manuscript.

\section{References}

1. Belkaid Y, Hand TW. Role of the Microbiota in Immunity and Inflammation. Cell 2014;157:121-41. https://doi.org/10.1016/j.cell.2014.03.011.

2. Ahern PP, Maloy KJ. Understanding immune-microbiota interactions in the intestine. Immunology 2020;159:4-14. https://doi.org/10.1111/imm.13150.

3. Ferreira RBR, Antunes LCM, Finlay BB. Should the Human Microbiome Be Considered When Developing Vaccines? PLOS Pathog 2010;6:e1001190. https://doi.org/10.1371/journal.ppat.1001190.

4. Zimmermann $P$, Curtis $N$. The influence of the intestinal microbiome on vaccine responses. Vaccine 2018;36:4433-9. https://doi.org/10.1016/j.vaccine.2018.04.066.

5. Hall JA, Bouladoux N, Sun CM, Wohlfert EA, Blank RB, Zhu Q, et al. Commensal DNA Limits Regulatory T Cell Conversion and Is a Natural Adjuvant of Intestinal Immune Responses. Immunity 2008;29:637-49. https://doi.org/10.1016/j.immuni.2008.08.009.

6. Norton EB, Lawson LB, Freytag LC, Clements JD. Characterization of a Mutant Escherichia coli HeatLabile Toxin, LT(R192G/L211A), as a Safe and Effective Oral Adjuvant. Clin Vaccine Immunol 2011;18:546-51. https://doi.org/10.1128/CVI.00538-10. 
7. Oh JZ, Ravindran R, Chassaing B, Carvalho FA, Maddur MS, Bower M, et al. TLR5-Mediated Sensing of Gut Microbiota Is Necessary for Antibody Responses to Seasonal Influenza Vaccination. Immunity 2014;41:478-92. https://doi.org/10.1016/j.immuni.2014.08.009.

8. Collins N, Belkaid Y. Do the microbiota influence vaccines and protective immunity to pathogens? Engaging our endogenous adjuvants. Cold Spring Harb Perspect Biol 2018;10:a028860.

9. Kukkonen K, Nieminen T, Poussa T, Savilahti E, Kuitunen M. Effect of probiotics on vaccine antibody responses in infancy - a randomized placebo-controlled double-blind trial. Pediatr Allergy Immunol 2006;17:416-21. https://doi.org/10.1111/j.1399-3038.2006.00420.x.

10. Soh SE, Ong DQR, Gerez I, Zhang X, Chollate P, Shek LP-C, et al. Effect of probiotic supplementation in the first 6 months of life on specific antibody responses to infant Hepatitis $B$ vaccination. Vaccine 2010;28:2577-9. https://doi.org/10.1016/j.vaccine.2010.01.020.

11. Trama AM, Moody MA, Alam SM, Jaeger FH, Lockwood B, Parks R, et al. HIV-1 Envelope gp41 Antibodies Can Originate from Terminal Ileum B Cells that Share Cross-Reactivity with Commensal Bacteria. Cell Host Microbe 2014;16:215-26. https://doi.org/10.1016/j.chom.2014.07.003.

12. Williams WB, Han Q, Haynes BF. Cross-reactivity of HIV vaccine responses and the microbiome: Curr Opin HIV AIDS 2018;13:9-14. https://doi.org/10.1097/COH.0000000000000423.

13. Slack E, Balmer ML, Macpherson AJ. B cells as a critical node in the microbiota-host immune system network. Immunol Rev 2014;260:50-66. https://doi.org/10.1111/imr.12179.

14. Smith K, McCoy KD, Macpherson AJ. Use of axenic animals in studying the adaptation of mammals to their commensal intestinal microbiota. Semin Immunol 2007;19:59-69. https://doi.org/10.1016/j.smim.2006.10.002.

15. Kim M, Qie Y, Park J, Kim CH. Gut Microbial Metabolites Fuel Host Antibody Responses. Cell Host Microbe 2016;20:202-14. https://doi.org/10.1016/j.chom.2016.07.001.

16. Wu W, Sun M, Chen F, Cao AT, Liu H, Zhao Y, et al. Microbiota metabolite short-chain fatty acid acetate promotes intestinal IgA response to microbiota which is mediated by GPR43. Mucosal Immunol 2017;10:946-56. https://doi.org/10.1038/mi.2016.114.

17. Kubinak JL, Stephens WZ, Soto R, Petersen C, Chiaro T, Gogokhia L, et al. MHC variation sculpts individualized microbial communities that control susceptibility to enteric infection. Nat Commun 2015;6:8642. https://doi.org/10.1038/ncomms9642.

18. Fadlallah J, Kafsi HE, Sterlin D, Juste C, Parizot C, Dorgham K, et al. Microbial ecology perturbation in human IgA deficiency. Sci Transl Med 2018;10. https://doi.org/10.1126/scitransImed.aan1217.

19. Hapfelmeier S, Lawson MAE, Slack E, Kirundi JK, Stoel M, Heikenwalder M, et al. Reversible microbial colonization of germ-free mice reveals the dynamics of IgA immune responses. Science 2010;328:1705-9. https://doi.org/10.1126/science.1188454.

20. Lécuyer E, Rakotobe S, Lengliné-Garnier H, Lebreton C, Picard M, Juste $C$, et al. Segmented Filamentous Bacterium Uses Secondary and Tertiary Lymphoid Tissues to Induce Gut IgA and Specific T Helper 17 Cell Responses. Immunity 2014;40:608-20. https://doi.org/10.1016/j.immuni.2014.03.009. 
21. Lindner C, Wahl B, Föhse L, Suerbaum S, Macpherson AJ, Prinz I, et al. Age, microbiota, and T cells shape diverse individual IgA repertoires in the intestine. J Exp Med 2012;209:365-77. https://doi.org/10.1084/jem.20111980.

22. Macpherson AJ, Yilmaz B, Limenitakis JP, Ganal-Vonarburg SC. IgA Function in Relation to the Intestinal Microbiota. Annu Rev Immunol 2018;36:359-81. https://doi.org/10.1146/annurevimmunol-042617-053238.

23. Pabst O, Hornef M. Gut Microbiota: A Natural Adjuvant for Vaccination. Immunity 2014;41:349-51. https://doi.org/10.1016/j.immuni.2014.09.002.

24. Gaucher D, Therrien R, Kettaf N, Angermann BR, Boucher G, Filali-Mouhim A, et al. Yellow fever vaccine induces integrated multilineage and polyfunctional immune responses. $J$ Exp Med 2008;205:3119-31. https://doi.org/10.1084/jem.20082292.

25. Querec TD, Akondy RS, Lee EK, Cao W, Nakaya HI, Teuwen D, et al. Systems biology approach predicts immunogenicity of the yellow fever vaccine in humans. Nat Immunol 2009;10:116-25. https://doi.org/10.1038/ni.1688.

26. Ehrenberg PK, Shangguan S, Issac B, Alter G, Geretz A, Izumi T, et al. A vaccine-induced gene expression signature correlates with protection against SIV and HIV in multiple trials. Sci Transl Med 2019;11. https://doi.org/10.1126/scitranslmed.aaw4236.

27. Rechtien A, Richert L, Lorenzo H, Martrus G, Hejblum B, Dahlke C, et al. Systems Vaccinology Identifies an Early Innate Immune Signature as a Correlate of Antibody Responses to the Ebola Vaccine rVSV-ZEBOV. Cell Rep 2017;20:2251-61. https://doi.org/10.1016/j.celrep.2017.08.023.

28. Nakaya HI, Wrammert J, Lee EK, Racioppi L, Marie-Kunze S, Haining WN, et al. Systems biology of vaccination for seasonal influenza in humans. Nat Immunol 2011;12:786-95. https://doi.org/10.1038/ni.2067.

29. Furman D, Jojic V, Kidd B, Shen-Orr S, Price J, Jarrell J, et al. Apoptosis and other immune biomarkers predict influenza vaccine responsiveness. Mol Syst Biol 2014;9:659-659. https://doi.org/10.1038/msb.2013.15.

30. Parvandeh S, Poland GA, Kennedy RB, McKinney BA. Multi-Level Model to Predict Antibody Response to Influenza Vaccine Using Gene Expression Interaction Network Feature Selection. Microorganisms 2019;7. https://doi.org/10.3390/microorganisms7030079.

31. Team H-CSP, Consortium H-I. Multicohort analysis reveals baseline transcriptional predictors of influenza vaccination responses. Sci Immunol 2017;2. https://doi.org/10.1126/sciimmunol.aal4656.

32. Tsang JS, Schwartzberg PL, Kotliarov Y, Biancotto A, Xie Z, Germain RN, et al. Global analyses of human immune variation reveal baseline predictors of post-vaccination responses. Cell 2014;157:499-513. https://doi.org/10.1016/j.cell.2014.03.031.

33. Plotkin SA. Correlates of Protection Induced by Vaccination. Clin Vaccine Immunol 2010;17:105565. https://doi.org/10.1128/CVI.00131-10.

34. Tsang JS. Utilizing population variation, vaccination, and systems biology to study human immunology. Trends Immunol 2015;36:479-93. https://doi.org/10.1016/j.it.2015.06.005. 
35. A. S, Bittencourt a S. FastQC: a quality control tool for high throughput sequence data 2010.

36. Bolger AM, Lohse M, Usadel B. Trimmomatic: a flexible trimmer for Illumina sequence data. Bioinforma Oxf Engl 2014;30:2114-20. https://doi.org/10.1093/bioinformatics/btu170.

37. Schloss PD, Westcott SL, Ryabin T, Hall JR, Hartmann M, Hollister EB, et al. Introducing mothur: opensource, platform-independent, community-supported software for describing and comparing microbial communities. Appl Environ Microbiol 2009;75:7537-41.

https://doi.org/10.1128/AEM.01541-09.

38. McDonald D, Price MN, Goodrich J, Nawrocki EP, DeSantis TZ, Probst A, et al. An improved Greengenes taxonomy with explicit ranks for ecological and evolutionary analyses of bacteria and archaea. ISME J 2012;6:610-8. https://doi.org/10.1038/ismej.2011.139.

39. Cosma A, Bühler S, Nagaraj R, Staib C, Hammarin A-L, Wahren B, et al. Neutralization Assay Using a Modified Vaccinia Virus Ankara Vector Expressing the Green Fluorescent Protein Is a HighThroughput Method To Monitor the Humoral Immune Response against Vaccinia Virus. Clin Diagn Lab Immunol 2004;11:406-10. https://doi.org/10.1128/CDLI.11.2.406-410.2004.

40. Earl PL, Americo JL, Moss B. Development and Use of a Vaccinia Virus Neutralization Assay Based on Flow Cytometric Detection of Green Fluorescent Protein. J Virol 2003;77:10684-8. https://doi.org/10.1128/JVI.77.19.10684-10688.2003.

41. García F, Bernaldo de Quirós JCL, Gómez CE, Perdiguero B, Nájera JL, Jiménez V, et al. Safety and immunogenicity of a modified pox vector-based HIV/AIDS vaccine candidate expressing Env, Gag, Pol and Nef proteins of HIV-1 subtype B (MVA-B) in healthy HIV-1-uninfected volunteers: A phase I clinical trial (RISVAC02). Vaccine 2011;29:8309-16. https://doi.org/10.1016/j.vaccine.2011.08.098.

42. Irizarry RA, Bolstad BM, Collin F, Cope LM, Hobbs B, Speed TP. Summaries of Affymetrix GeneChip probe level data. Nucleic Acids Res 2003;31:e15. https://doi.org/10.1093/nar/gng015.

43. Huber W, von Heydebreck A, Sültmann H, Poustka A, Vingron M. Variance stabilization applied to microarray data calibration and to the quantification of differential expression. Bioinforma Oxf Engl 2002;18 Suppl 1:S96-104. https://doi.org/10.1093/bioinformatics/18.suppl_1.s96.

44. Bolstad BM, Irizarry RA, Astrand M, Speed TP. A comparison of normalization methods for high density oligonucleotide array data based on variance and bias. Bioinforma Oxf Engl 2003;19:18593. https://doi.org/10.1093/bioinformatics/19.2.185.

45. Larsen JM. The immune response to Prevotella bacteria in chronic inflammatory disease. Immunology 2017;151:363-74. https://doi.org/10.1111/imm.12760.

46. Shibagaki N, Suda W, Clavaud C, Bastien P, Takayasu L, lioka E, et al. Aging-related changes in the diversity of women's skin microbiomes associated with oral bacteria. Sci Rep 2017;7:1-10. https://doi.org/10.1038/s41598-017-10834-9.

47. King CH, Desai H, Sylvetsky AC, LoTempio J, Ayanyan S, Carrie J, et al. Baseline human gut microbiota profile in healthy people and standard reporting template. Microbiology; 2018. https://doi.org/10.1101/445353. 
48. Le Bastard Q, Ward T, Sidiropoulos D, Hillmann BM, Chun CL, Sadowsky MJ, et al. Fecal microbiota transplantation reverses antibiotic and chemotherapy-induced gut dysbiosis in mice. Sci Rep 2018;8. https://doi.org/10.1038/s41598-018-24342-x.

49. Rinninella E, Raoul P, Cintoni M, Franceschi F, Miggiano GAD, Gasbarrini A, et al. What is the Healthy Gut Microbiota Composition? A Changing Ecosystem across Age, Environment, Diet, and Diseases. Microorganisms 2019;7. https://doi.org/10.3390/microorganisms7010014.

50. Biagi E, Nylund L, Candela M, Ostan R, Bucci L, Pini E, et al. Through Ageing, and Beyond: Gut Microbiota and Inflammatory Status in Seniors and Centenarians. PLoS ONE 2010;5. https://doi.org/10.1371/journal.pone.0010667.

51. Huda MN, Lewis Z, Kalanetra KM, Rashid M, Ahmad SM, Raqib R, et al. Stool Microbiota and Vaccine Responses of Infants. Pediatrics 2014;134:e362-72. https://doi.org/10.1542/peds.2013-3937.

52. Levine MM. Immunogenicity and efficacy of oral vaccines in developing countries: lessons from a live cholera vaccine. BMC Biol 2010;8:129. https://doi.org/10.1186/1741-7007-8-129.

53. Magwira CA, Taylor MB. Composition of gut microbiota and its influence on the immunogenicity of oral rotavirus vaccines. Vaccine 2018;36:3427-33. https://doi.org/10.1016/j.vaccine.2018.04.091.

54. Harris VC, Armah G, Fuentes S, Korpela KE, Parashar U, Victor JC, et al. Significant Correlation Between the Infant Gut Microbiome and Rotavirus Vaccine Response in Rural Ghana. J Infect Dis 2017;215:34-41. https://doi.org/10.1093/infdis/jiw518.

55. Burkhardt AL, Brunswick M, Bolen JB, Mond JJ. Anti-immunoglobulin stimulation of B lymphocytes activates src-related protein-tyrosine kinases. Proc Natl Acad Sci USA 1991;88:7410-4.

56. Saijo K, Schmedt C, Su I-hsin, Karasuyama H, Lowell CA, Reth M, et al. Essential role of Src-family protein tyrosine kinases in NF-KB activation during B cell development. Nat Immunol 2003;4:274-9. https://doi.org/10.1038/ni893.

57. Musa YR, Boller S, Puchalska M, Grosschedl R, Mittler G. Comprehensive Proteomic Investigation of Ebf1 Heterozygosity in Pro-B Lymphocytes Utilizing Data Independent Acquisition. J Proteome Res 2018;17:76-85. https://doi.org/10.1021/acs.jproteome.7b00369.

\section{Figures}




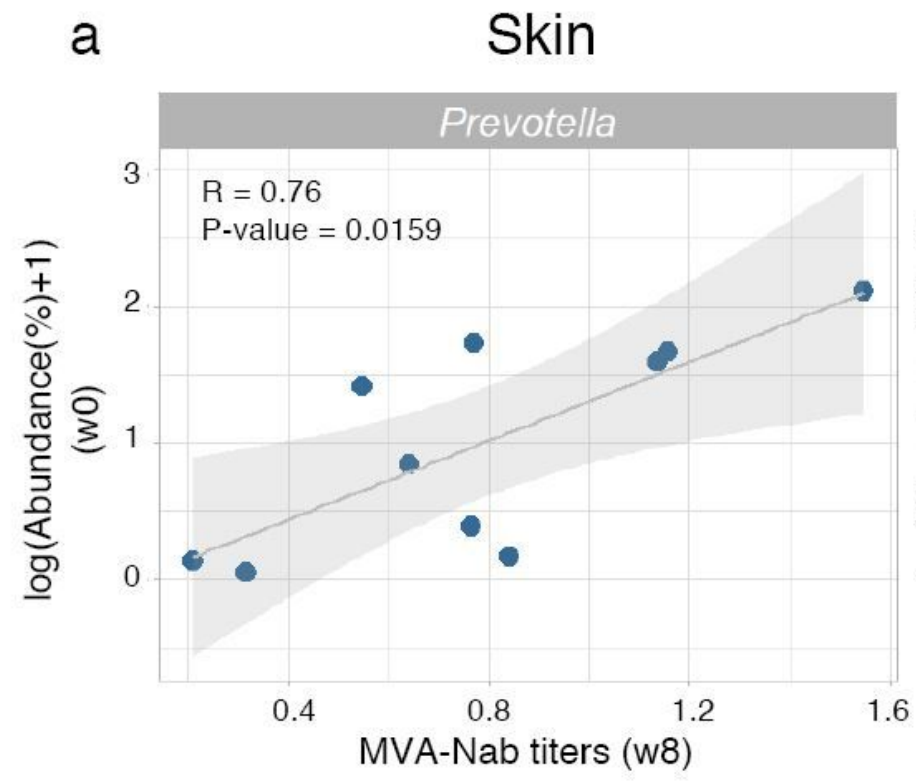

b Stool

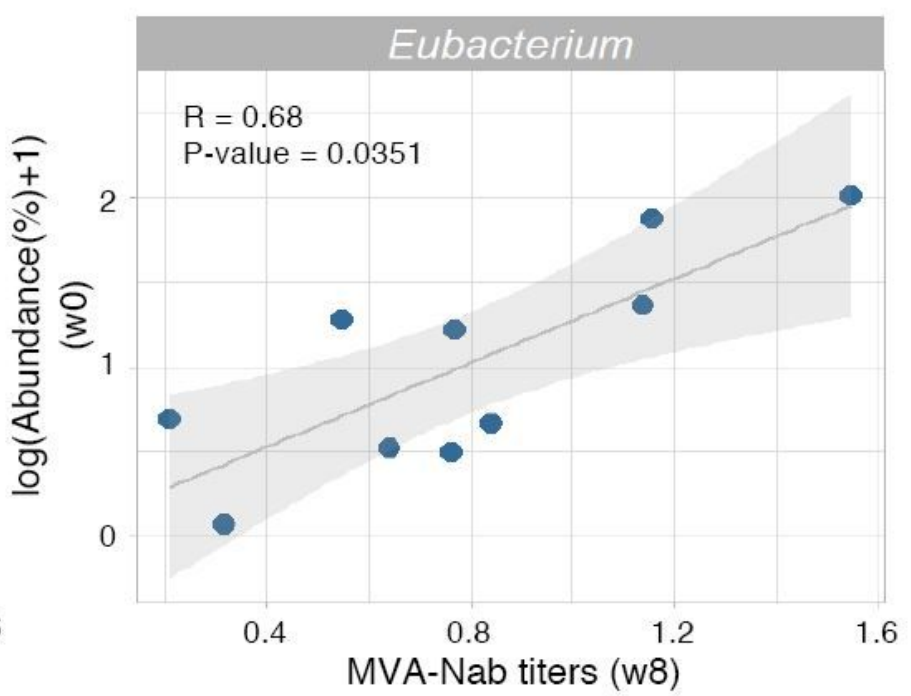

\section{Figure 1}

Microbial abundance before vaccination is correlated with MVA-Nab responses (a) Abundance of Prevotella in skin and (b) abundance of Eubacterium in stool are correlated with MVA-specific neutralizing antibody titers at w8 (log1/EC50). Spearman rank sum test was applied with a P-value $<0.05$. All genera were filtered by a minimum median abundance of $0.1 \%$ across the samples. 


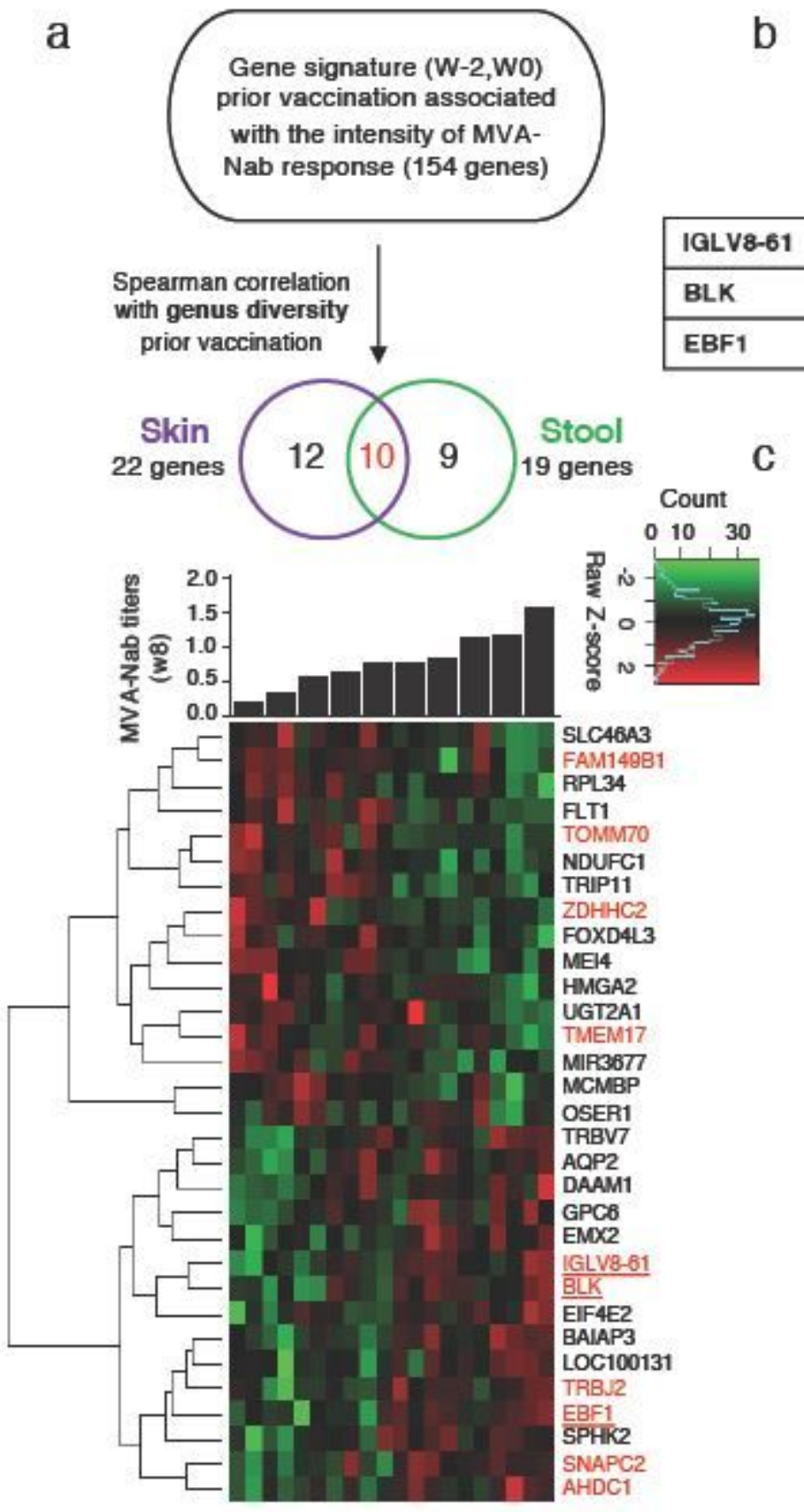

b

\begin{tabular}{|l|c|c|c|c|}
\cline { 2 - 3 } \multicolumn{1}{c|}{} & \multicolumn{2}{c|}{$\begin{array}{c}\text { Prevotella } \\
\text { abundance }\end{array}$} & \multicolumn{2}{c|}{$\begin{array}{c}\text { Eubacterium } \\
\text { abundance }\end{array}$} \\
\cline { 2 - 5 } \multicolumn{1}{c|}{} & $\mathbf{P}$ & P-value & R & P-value \\
\hline IGLV8-61 & -0.64 & 0.0035 & -0.54 & 0.0145 \\
\hline BLK & -0.66 & 0.0014 & -0.56 & 0.0105 \\
\hline EBF1 & -0.58 & 0.0078 & -0.58 & 0.0078 \\
\hline
\end{tabular}

C
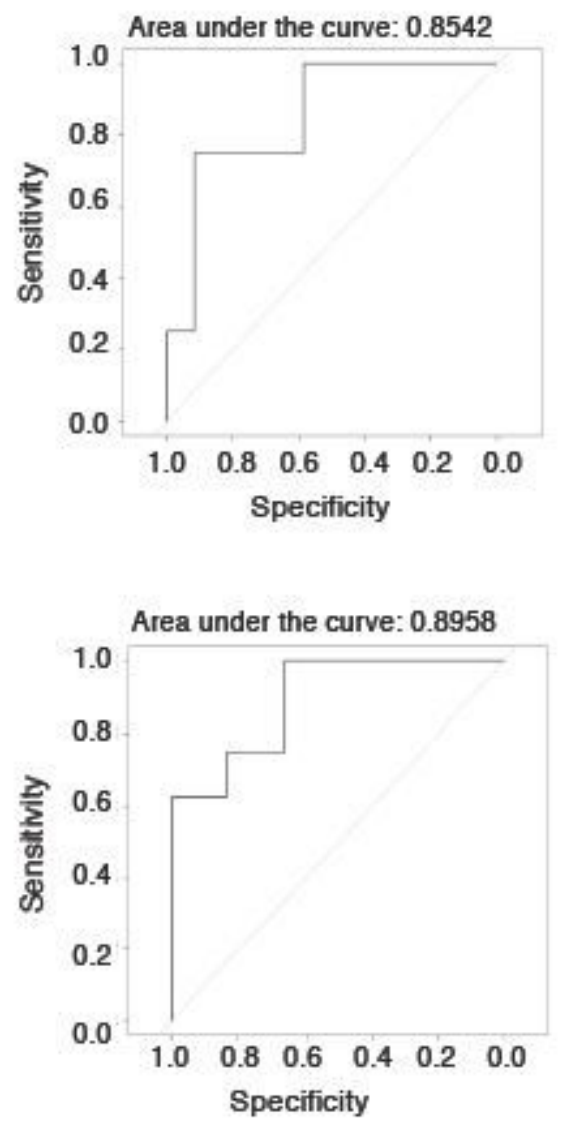

\section{Figure 2}

Blood gene expression combined with host microbiota before vaccination shapes MVA-B responses (a) Investigation of the blood gene expression ( $w-2, w 0)$ correlated with MVA-Nab response (w8) and host genus diversity (w0), for skin (green) and stool (purple). The Spearman correlation test was applied with $P<0.05$ defined as statistically significant. The heatmap shows the expression profile of the 22 (purple) and 19 (green) genes correlated respectively with skin and stool, according to MVA-Nab response intensity from lowest to highest responders. The 10 common genes are colored in red. The color-gradient from green $(-2$, low $)$ to red $(2$, high) indicates the intensity of gene expression. Among the genes 
correlated with both skin and stool, three genes are underlined because they are involved in B cell functions. (b) Table shows the significant correlation coefficients and P-values for each of the three genes with the abundance of the genus correlated with MVA-Nab response. (c) ROC curves show the specificity and the sensitivity of the logistic regression models, i.e., the proportion of correctly predicted responders and nonresponders, respectively. The logistic regression is based on the expression of the minimal gene signature (IGLV8, EBF1, and BLK) and the abundance of Eubacterium and Prevotella, respectively, in stool and skin.

\section{Supplementary Files}

This is a list of supplementary files associated with this preprint. Click to download.

- TableGenesFunctions.pdf

- manuscriptmicrobiome20072020supplfigures1.pdf 